\title{
Global Stability Analysis of Logistically Grown SIR Model with Loss of Immunity, Inhibitory Effect, Crowding Effect and its Protection Measure
}

\author{
Uttam Ghosh* , Susmita Sarkar \\ Department of Applied Mathematics, University of Calcutta, Kolkata, India \\ *E-mail: uttam_math@yahoo.co.in
}

Received: 12 December 2016; revised: 30 March 2018; accepted: 04 April 2018; published online: 30 May 2018

\begin{abstract}
In this paper we have considered an SIR model with logistically grown susceptible in which the rate of incidence is directly affected by the inhibitory factors of both susceptible and infected populations and the protection measure for the infected class. Permanence of the solutions, global stability and bifurcation analysis in the neighborhood of equilibrium points has been investigated here. The Center manifold theory is used to find the direction of bifurcations. Finally numerical simulation is carried out to justify the theoretical findings.
\end{abstract}

Key words: inhibition effect, Hopf bifurcation, logistic growth rate, loss immunity, normal forms, center manifold theory

\section{INTRODUCTION}

In recent years the public health planners have been using mathematical models to study the dynamics of different epidemic diseases. This branch is named mathematical epidemiology. In this branch models are formulated using the differential and difference equations[1-5]. Karmack and McKendrick [6] first formulated the SIS and SIR models to study the epidemiological dynamics (infection dynamics). In studying the SIR model the populations are divided into three classes: susceptible $(S)$, infected $(I)$ and recovered $(R)$ class. The important factors on which the infection dynamics depend are: (i) birth rate of the susceptible populations which may follow constant, exponential or logistic growth, (ii) normal death rate of the populations, (iii) rate of incidence of infection among the susceptible populations and (iv) recovery of infections and then loss of immunity. The mathematics of such dynamics are known from references [7-11]. In most of the articles researchers considered the constant birth rates of the susceptible populations [12-17], which is not realistic in some populations. So the authors in [18-20] considered exponential/logistic growth rate of the susceptible populations.

On the other hand authors considered several types of incidence of infection among the susceptibles populations. In case of standard incidence ( $p S I)$, the increase of susceptibles increase the number of individuals who become infected per unit time. This is not realistic: modification of this type of rate of incidence was necessary. Some researchers use modified incidence rates, e.g. the bi-linear incidence rate, saturated incidence rate, etc. [15, 21-24]. Similarly some authors [25-29] used the rate of incidence in the form $\frac{a S I}{1+b I}$. In this form of incidence the increase in the number of infected population increases the incidence term and it ultimately becomes proportional to the number of susceptible populations. Here a very high portion of the susceptible population is infected and causes a slowing of new infection. This type of effect is termed as the "crowing effect" or "protection measure". The term like $1+b I$ in the denominator of the incidence term measures the inhibition effect from the behavioral change of the 
susceptible individuals when the number of infectious individuals increases or from the crowding effect of the infective individuals [30]. Kar and Mondal [25] used the saturation effect in the form $\frac{p S I}{1+\alpha S}$, where $\alpha$ was defined as the half saturation constant. Here with the increase of the susceptible population the incidence term will increase and it ultimately becomes proportional to the number of infected individuals. This type of infection may be termed as the "psychological effects" because in the initial stage the number of infective is small, people may ignore the epidemic but there may be lots of effective contacts between the infective individuals and susceptibles, the number of infective individuals will increase and the protection measure will be taken into consideration [21].

In this paper we have considered a SIR model with logistic growth rate of the susceptibles where the rate of infection is directly affected by the inhibitory effect, crowding affect and protection measure. Here we are considering the Boddington and DeAngelis [31-34] type incidence rate $\left(\frac{p S I}{1+\alpha S+\beta I}\right)$ previously used by the references $[21,31,32]$. This type of saturation is a combination of all the previous effects. Our model in the present paper is an extension of the models from [17-21], where the authors considered the same rate of incidence but with different birth rate of the susceptible populations.

The paper is organized as follows: in Section II we have formulated the model, in Section III we describe the boundedness and permanence of the solutions. Local and global stability analysis of the equilibrium points is described in Section IV and Hopf bifurcation criterion is analyzed in Section V. In Section VI we used the Center manifold theory to investigate the direction bifurcations of the solutions about the equilibrium points. Numerical simulations are given in Section VII and finally some conclusions are given in Section VIII.

\section{MODEL FORMULATION}

Let $S(t), I(t)$ and $R(t)$ be the number of susceptible, infected and recovered individuals at time $t$. Here we consider the model in which the birth rate of the susceptible is a logistic type and the rate of infection is a saturation type and directly affected by the inhibitory factors, crowding effect and a protection measure. Incorporating all the assumptions the governing differential equation of the proposed model can be written in the following form

$$
\left\{\begin{array}{l}
\frac{d S}{d t}=r S\left(1-\frac{S}{k}\right)-\frac{p S I}{1+\alpha S+\beta I}-d S+\gamma R \\
\frac{d I}{d t}=\frac{p S I}{1+\alpha S+\beta I}-(d+\mu) I \\
\frac{d R}{d t}=\mu I-(d+\gamma) R,
\end{array}\right.
$$

where: $r$ - intrinsic growth rate of the susceptible class, $k$ - carrying capacity, $p$ - the rate of infection, $\alpha$ and $\beta$ - the parameter that measures the inhibitory factors may be social awareness of both the populations or crowding effect and protection measure of the infected populations, respectively, $d$ - the natural death of the population, $\gamma$-rate at which the recovered class loses immunity and becomes susceptible, $\mu$ - rate at which the infected individuals recovered.

In the model, if $\alpha$ and $\beta$ both increase then the term $\frac{p S I}{1+\alpha S+\beta I}$ will decrease i.e. lesser number of susceptible individual will be infected. Thus $\alpha$ and $\beta$ play an important role in the model. Here all the constants considered in the model are non-negative.

\section{BOUNDEDNESS AND PERMANENCE OF THE SOLUTIONS}

In this section we shall investigate the boundedness and permanence of the solution of the system (1). Theorems 1-3 provide the conditions of boundedness and Theorem 4 provides the conditions of permanence.

Theorem 1. All the feasible solutions of the system (1) are bounded and enter the region

$\Omega=\left\{(S, I, R) \in R^{3}, 0 \leq S+I+R \leq r k / d\right\}$.

Proof. Let $W=S+I+R$

$$
\begin{gathered}
\therefore \frac{d W}{d t}=\frac{d S}{d t}+\frac{d I}{d t}+\frac{d R}{d t} \leq r k-d(S+I+R) \\
\therefore \frac{d W}{d t}+W d \leq r k .
\end{gathered}
$$

Integrating both sides we get, $W(t) \leq \frac{r k}{d}+W_{0} e^{-d t} \rightarrow$ $\frac{r k}{d}$ as $t \rightarrow \infty$, where $W_{0}$ is the initial condition. Hence all the feasible solutions enter in the region $\Omega$.

Theorem 2. If $S(t), I(t)$ and $R(t)$ be the solution of the system (1) then in the region $S d \geq R \gamma$ the following hold

$$
\left\{\begin{array}{l}
\limsup _{t \rightarrow \infty} S(t) \leq k \\
\limsup _{t \rightarrow \infty} I(t) \leq \frac{p k}{\beta(d+\mu)} \\
\limsup _{t \rightarrow \infty} R(t) \leq \frac{p k \mu}{\beta(d+\mu)}
\end{array}\right.
$$

Proof. From the first equation of the system (1) we have $\frac{d S}{d t} \leq r S\left(1-\frac{S}{k}\right)$ in the region $S d \geq R \gamma$. Let $\bar{S}$ be the solution of the following initial valued problem $\frac{d \bar{S}}{d t}=$ $r \bar{S}\left(1-\frac{\bar{S}}{k}\right), \bar{S}(0)=x(0)$. By standard comparison principal, we have $S(t) \leq \bar{S}(t)$ for all $t \in[0,+\infty]$ which follows,

$$
\limsup _{t \rightarrow \infty} S(t) \leq \limsup _{t \rightarrow \infty} \bar{S}(t)=k
$$


From the second equation of the system (1), using (2) we have $\frac{d I}{d t} \leq\left\{\frac{p k}{\beta}-(\mu+d) I\right\}$, which implies

$$
\limsup _{t \rightarrow \infty} I(t) \leq \frac{p k}{\beta(d+\mu)}
$$

Similarly the third equation of (1) along with (2) and (3) follows

$$
\limsup _{t \rightarrow \infty} R(t) \leq \frac{p k \mu}{\beta(d+\mu)}
$$

Theorem 3. If $S(t), I(t)$ and $R(t)$ are the solution of the system (1) and $r>d$ then

$$
\left\{\begin{array}{l}
\liminf _{t \rightarrow \infty} S(t) \geq k\left(1-\frac{d}{r}\right) \\
\liminf _{t \rightarrow \infty} I(t) \geq \frac{p k\left(1-\frac{d}{r}\right)}{c(d+\mu)\left(1+k\left(1-\frac{d}{r}\right)\right)} \\
\liminf _{t \rightarrow \infty} R(t) \geq \frac{p \mu k\left(1-\frac{d}{r}\right)}{c(d+\gamma)(d+\mu)\left(1+k\left(1-\frac{d}{r}\right)\right)}
\end{array}\right.
$$

where $c=\max \{1, \alpha, \beta\}$.

Proof. The first equation of the system (1) can be written in the form $\frac{d S}{d t} \geq r S\left(1-\frac{S}{k}\right)-d S$. Then $\hat{S}=\frac{k(1-d / r)}{1+c e^{(-(r-d) t)}}$ satisfies the following differential equation

$$
\frac{d \hat{S}}{d t}=r \hat{S}\left(1-\frac{\hat{S}}{k}\right)-d \hat{S}
$$

$c$ is arbitrary constant.

This implies, $\liminf _{t \rightarrow \infty} S(t) \geq \liminf _{t \rightarrow \infty} \hat{S(t)}=k(1-d / r)$. Proceeding in the same manner with other two equations of (1) it can be easily shown that

$$
\begin{gathered}
\liminf _{t \rightarrow \infty} I(t) \geq \frac{p k\left(1-\frac{d}{r}\right)}{c(d+\mu)\left(1+k\left(1-\frac{d}{r}\right)\right)} \\
\liminf _{t \rightarrow \infty} R(t) \geq \frac{p \mu k\left(1-\frac{d}{r}\right)}{c(d+\gamma)(d+\mu)\left(1+k\left(1-\frac{d}{r}\right)\right)}
\end{gathered}
$$

where $c=\max \{1, \alpha, \beta\}$.

Thus the above three theorems show that solutions of system (1) are bounded from both below and above. Permanence of the solution of (1) can be determined from the following Theorem 4.

Definition of permanence [35]: The system (1) is said to be permanent if there exist $M$ and $m, 0 \leq m \leq M$ such that for all solutions of (1) the following conditions are satisfied

$$
\begin{gathered}
\operatorname{Min}\left\{\liminf _{\rightarrow \infty} S(t), \liminf _{t \rightarrow \infty} I(t), \liminf _{t \rightarrow \infty} R(t)\right\} \geq m, \\
\operatorname{Max}\left\{\limsup _{t \rightarrow \infty} S(t), \limsup _{t \rightarrow \infty} I(t), \limsup _{t \rightarrow \infty} R(t)\right\} \leq M .
\end{gathered}
$$

Theorem 4. The system (1) is permanent inside the plane $d S \geq \gamma R$.

Proof. The theorem immediately follows if we choose

$$
\left.\begin{array}{c}
M=\operatorname{Max}\left\{k, \frac{p k}{\beta(d+\mu)}, \frac{p k \mu}{\beta(d+\mu)}\right\} \\
\text { and } \operatorname{Min}\left\{k\left(1-\frac{d}{r}\right), \frac{p k\left(1-\frac{d}{r}\right)}{c(d+\mu)\left(1+k\left(1-\frac{d}{r}\right)\right)},\right. \\
\\
c(d+\gamma)(d+\mu)\left(1+k\left(1-\frac{d}{r}\right)\right)
\end{array}\right\}
$$

where $c$ is already defined. Then all conditions of the definition of permanence are satisfied.

This theorem implies that the system (1) is permanent if the number of deaths of susceptible individuals at any time is the same or higher than the number of recovered individuals who have again become susceptible.

\section{STABILITY ANALYSIS OF THE MODEL}

There are three equilibrium points in our considered model, namely the trivial equilibrium $(0,0,0)$, the disease free equilibrium $\left(S_{0}, 0,0\right)$, and the endemic equilibrium point $\left(S_{1}, I_{1}, R_{1}\right)$, where $S_{0}=k\left(1-\frac{d}{r}\right), I_{0}=0, R_{0}=0$, $I_{1}=\frac{\left(1+\alpha S_{1}\right)}{\beta}\left(R_{01}-1\right), R_{1}=\frac{\mu I_{1}}{d+\mu}$ and $R_{01}=$ $\frac{p S_{1}}{\left(1+\alpha S_{1}\right)(d+\mu)}$ and $S_{1}$ is positive root of the algebraic equation

$$
S^{2}+a_{1} S-a_{2}=0
$$

were $a_{1}=\frac{k}{\beta r}\left\{\frac{a p}{d+\mu}+\beta d-(\beta r+a \alpha)\right\}, a_{2}=$ $\frac{a k}{\beta r}, a=\frac{d^{2}+d \mu+d \gamma}{d+\gamma}$ and $S_{1}=\frac{-a_{1}+\sqrt{a_{1}^{2}+4 a_{2}}}{2}$.

Expression of $I_{1}$ shows that the equilibrium point will exist only when $R_{01}>1$. The basic reproduction number of the considered model has been calculated in the form

$$
R_{0}=\frac{p k\left(1-\frac{d}{r}\right)}{(d+\mu)\left(1+k\left(1-\frac{d}{r}\right) \alpha\right)}
$$

by compartmental method [38]. Here the variational matrix corresponding to the model (1) is 


$$
J(S, I, R)=\left(\begin{array}{ccc}
r\left(1-\frac{2 S}{k}\right)-d-\frac{p I(1+\beta I)}{(1+\alpha S+\beta I)^{2}} & \frac{p I(1+\beta I)}{(1+\alpha S+\beta I)^{2}} & 0 \\
-\frac{p S(1+\alpha S)}{(1+\alpha S+\beta I)^{2}} & \frac{p S(1+\alpha S)}{(1+\alpha S+\beta I)^{2}}-(d+\mu) & \mu \\
\gamma & 0 & -(d+\gamma)
\end{array}\right)
$$

Theorem 5. If $r<d$ then the trivial equilibrium point $(0,0,0)$ is asymptotically stable.

Proof. The characteristic equation for the equilibrium point $(0,0,0)$ is

$$
\left|\begin{array}{ccc}
r-d-\lambda & 0 & 0 \\
0 & -(d+\mu)-\lambda & \mu \\
\gamma & 0 & -(d+\gamma)-\lambda
\end{array}\right|=0
$$

Roots of the above equations are $r-d,-(d+\mu)$ and $-(d+\gamma)$. Since if $r<d$ then all roots are negative and consequently this equilibrium point is asymptotically stable. On the other hand, if $r>d$ then one of the three roots is positive and the other two are negative. Consequently, the equilibrium point is a saddle point and the solution in the neighbourhood of this equilibrium point will be unstable in nature.

Theorem 6. If $r<d$ and $\frac{p r k}{(d+r k \alpha)(d+\mu)}<1$ then the trivial equilibrium point $(0,0,0)$ is globally asymptotically stable.

Proof. Consider the Lyapunov function

$$
L=I
$$

The sign of equality holds only when $I=0$.

Let $S_{1}=\{(S, I, R): \dot{L}=0\}=\{(S, I, R): I=0\}$ and $M$ be the largest invariant set in $S_{1}$. Then putting $I=0$ in equations of (1), integrating and using the conditions stated in the theorem we get, $R \rightarrow 0$ and $S \rightarrow 0$ as $t \rightarrow \infty$.

Thus the invariant set

$$
M=\{(0,0,0)\}
$$

is a singleton set. Hence it follows from Lasalle-Liapunov theory [36] that the equilibrium point $(0,0,0)$ is globally asymptotically stable. Hence the theorem follows.

In the next part we shall describe the stability of the disease free equilibrium point $\left(k\left(1-\frac{d}{r}\right), 0,0\right)$.

Theorem 7. If $r>d$ and $R_{0}<1$ then the disease free equilibrium point $\left(k\left(1-\frac{d}{r}\right), 0,0\right)$ is asymptotically stable.

Proof. The characteristic equation corresponding to this equilibrium point is

$$
\left|\begin{array}{ccc}
-(r-d-\lambda) & 0 & 0 \\
-\frac{p k\left(1-\frac{d}{r}\right)}{1+\alpha k\left(1-\frac{d}{r}\right)} & \frac{p k\left(1-\frac{d}{r}\right)}{1+\alpha k\left(1-\frac{d}{r}\right)}-(d+\mu)-\lambda & \mu \\
\gamma & 0 & -(d+\gamma)-\lambda
\end{array}\right|=0
$$

whose roots are $-(r-d),-(d+\gamma)$ and $\frac{p k\left(1-\frac{d}{r}\right)}{1+\alpha k\left(1-\frac{d}{r}\right)}-(d+\mu)=(d+\mu)\left(R_{0}-1\right)$. If $R_{0}<1$ then all the roots are negative and solution in the neighbourhood of this equilibrium point is asymptotically stable and if $R_{0}>1$ then one root is positive and the other two roots are negative and consequently the equilibrium point is unstable in nature.

Hence the theorem follows. 
We shall next discuss the global asymptotic stability of the disease free equilibrium point.

Theorem 8. If $r>d$ and $p<\frac{d(d+\mu)}{r k}$ then the disease free equilibrium point $\left(k\left(1-\frac{d}{r}\right), 0,0\right)$ is globally asymptotically stable.

Proof. To check the global asymptotically stability of disease free equilibrium point consider the Lyapunov function $L=I$ then

$$
\begin{aligned}
& \frac{d L}{d t}=\frac{d I}{d t}=\left\{\frac{p S}{1+\alpha S+\beta I}-(\mu+d)\right\} I \\
\leq & \{p S-(d+\mu)\} I \\
\leq & \left\{\frac{p r k}{d}-(d+\mu)\right\} I \leq 0 \text { if } p<\frac{d(d+\mu)}{r k} .
\end{aligned}
$$

Here also the sign of equality holds when $I=0$. Let the set $S_{1}=\{(S, I, R): \dot{L}=0\}=\{(S, I, R): I=0\}$ and $M$ be the largest invariant in $S_{1}$. Then from the set $S_{1}$ putting
$I=0$ in the system of equation (1) and integrating and using the given conditions we get, $R \rightarrow 0$, and $S \rightarrow k\left(1-\frac{d}{r}\right)$ as $t \rightarrow \infty$.

Thus $M=\left\{\left(k\left(1-\frac{d}{r}\right), 0,0\right)\right\}$ is a singleton set, hence it follows from the Lasalle-Liapunov theory [36] that the equilibrium point $\left(k\left(1-\frac{d}{r}\right), 0,0\right)$ is globally asymptotically stable.

Thus the disease free equilibrium point $\left(k\left(1-\frac{d}{r}\right), 0,0\right)$ is globally asymptotically stable up to a critical value of the rate of infection $(p)$, above which it fails to become globally asymptotically stable. In this section we shall describe the nature of the solution in the neighbourhood of the endemic equilibrium point $\left(S_{1}, I_{1}, R_{1}\right)$.

Theorem 9. The endemic equilibrium point $\left(S_{1}, I_{1}, R_{1}\right)$ is asymptotically stable if $R_{01}>1, R_{0}>1$ and $C_{1}, C_{2}, C_{3}, C_{1} C_{2}-C_{3}$ all are positive, where $C_{1}, C_{2}, C_{3}$ defined in the proof.

Proof. The characteristic equation corresponding to the endemic equilibrium point $\left(S_{1}, I_{1}, R_{1}\right)$ is

$$
\left|\begin{array}{ccc}
r S_{1}\left(1-\frac{2 S_{1}}{k}\right)-A-d-\lambda & A & 0 \\
-B & B-d-\mu-\lambda & \mu \\
\gamma & 0 & -(d+\gamma)-\lambda
\end{array}\right|=0
$$

where:

$$
A=\frac{p I_{1}\left(1+\beta I_{1}\right)}{\left(1+\alpha S_{1}+\beta I_{1}\right)^{2}}
$$

and

$$
B=\frac{p S_{1}\left(1+\alpha S_{1}\right)}{\left(1+\alpha S_{1}+\beta I_{1}\right)^{2}} .
$$

After simplifying the equation (9) we get

$$
\lambda^{3}+C_{1} \lambda^{2}+C_{2} \lambda+C_{3}=0
$$

where:

$$
\begin{aligned}
& C_{1}=2 d+\gamma+r\left(\frac{2 S_{1}}{k}-1\right)+A+\frac{(d+\mu) \beta I_{1}}{\left(1+\alpha S_{1}+\beta I_{1}\right)}, \\
& C_{2}=\left(d+\gamma+\frac{\left.(d+\mu) \beta I_{1}\right)}{\left(1+\alpha S_{1}+\beta I_{1}\right)}\right)\left(A+d+r\left(\frac{2 S_{1}}{k}-1\right)\right)+(d+\gamma) \frac{(d+\mu) \beta I_{1}}{\left(1+\alpha S_{1}+\beta I_{1}\right)}+A B, \\
& C_{3}=d(d+\mu+\gamma) A+\frac{(d+\gamma)(d+\mu) \beta I_{1}}{\left(1+\alpha S_{1}+\beta I_{1}\right)}\left(d+r\left(\frac{2 S_{1}}{k}-1\right)\right) .
\end{aligned}
$$


If $C_{1}, C_{2}, C_{3}$ and $C_{1} C_{2}-C_{3}$ are all positive then by Routh-Hurwitz criteria all roots have negative real part and consequently the solution in the neighbourhood of endemic equilibrium point is asymptotically stable. If one of $C_{1}, C_{3}$ failed to become positive then stability fails.

Global asymptotic stability of the endemic equilibrium point follows from the following theorem.

Theorem 10. The system (1) is globally asymptotically stable around the endemic equilibrium point $\left(S_{1}, I_{1}, R_{1}\right)$ in the region $\Omega_{1}=\left\{(S, I, R): 1<\frac{I_{1}}{I}<\frac{R_{1}}{R}<\frac{S_{1}}{S}\right\}$ when $R_{01}>1$.

Proof. To prove the global asymptotic stability we construct the positive Lyapunov function in the following form

$$
\begin{aligned}
& L(S, I, R)=\int_{S_{1}}^{S} \frac{S-S_{1}}{S} d S+\int_{I_{1}}^{I} \frac{I-I_{1}}{I} d I \\
+ & \int_{R_{1}}^{R} \frac{R-R_{1}}{R} d R, \text { then } \\
& \frac{d L}{d t}=\frac{S-S_{1}}{S} \frac{d S}{d t}+\frac{I-I_{1}}{I} \frac{d I}{d t}+\frac{R-R_{1}}{R} \frac{d R}{d t}= \\
= & -\frac{r\left(S-S_{1}\right)^{2}}{k}-\frac{p \alpha\left(S-S_{1}\right)\left(I S_{1}-S I_{1}\right)}{(1+\alpha S+\beta I)\left(1+\alpha S_{1}+\beta I_{1}\right)} \\
- & \frac{r\left(S-S_{1}\right)\left(R_{1} S-R S_{1}\right)}{S S_{1}}
\end{aligned}
$$$$
\begin{aligned}
& -\frac{p \beta\left(I-I_{1}\right)\left(I S_{1}-S I_{1}\right)}{(1+\alpha S+\beta I)\left(1+\alpha S_{1}+\beta I_{1}\right)} \\
& -\frac{\left.\mu\left(R-R_{1}\right)\left(R I_{1}-I R_{1}\right)\right)}{R R_{1}} .
\end{aligned}
$$

Since in the considered region $\Omega_{1}$ the function $\frac{d L}{d t}<0$, by Lasalle-Liapunov theory [36] the system (1) is globally asymptotically stable.

\section{HOPF BIFURCATIONS}

If the coefficients $C_{1}, C_{2}, C_{3}$ of the characteristic polynomial equation (10) satisfy the inequalities $C_{1}>0, C_{2}>$ $0, C_{3}>0$ and $C_{1} C_{2}-C_{3}>0$ then the equilibrium point is asymptotically stable. On the other hand, if the forth inequality fails, i.e. $C_{1} C_{2}-C_{3}=0$, then equation (10) has a pair of complex roots with zero real part. This may cause Hopf bifurcation if the transversality condition is satisfied.

Theorem 11. If $R_{01}>1, R_{0}>1, C_{1}>0, C_{2}>0$, $C_{3}>0, C_{1} C_{2}-C_{3}=0$ and the intrinsic growth rate $r$ passes through $r=r_{c}$ and the transversality conditions
$\left\{\frac{d\left(C_{1} C_{2}-C_{3}\right)}{d r}\right\}_{r=r_{c}} \neq 0$ is satisfied then the system (1) goes through Hopf bifurcation in the neighbourhood of the endemic equilibrium point $\left(S_{1}, I_{1}, R_{1}\right)$, where $r_{c}$ is defined in the proof.

Proof. If $C_{1}, C_{2}, C_{3}$ are positive and $C_{1} C_{2}-C_{3}=0$,occurs for $r=r_{c}$ then two roots of the equation will be purely imaginary and the other will be negative. Thus, for any $\epsilon>0$ there exists an interval containing $\left(r_{c}-\epsilon, r_{c}+\epsilon\right)$ such that for $r \in\left(r_{c}-\epsilon, r_{c}+\epsilon\right)$, the characteristic equation (10) cannot have real positive roots. For $r=r_{c}$ the equation (10) can be expressed as $\left(\lambda^{2}+C_{2}\right)\left(\lambda+C_{1}\right)=0$, roots of this equation are $\lambda_{1,2}= \pm i \sqrt{C_{2}}, \lambda_{3}=-C_{1}$. Thus for $r \in\left(r_{c}-\epsilon, r_{c}+\epsilon\right)$ the roots are in general of the form $\lambda_{1,2}=\alpha_{1} \pm i \beta_{1}, \lambda_{3}=-C_{1}$ where $\alpha_{1}\left(r_{c}\right)=0$.

For Hopf bifurcation to occur, the transversality condition $\operatorname{Real}\left(\frac{d \lambda_{1,2}}{d r}\right)_{r=r_{c}} \neq 0$ must satisfy.

Now putting $\lambda=\alpha_{1}+i \beta_{1}$ in equation (10) we get $\left(\alpha_{1}+i \beta_{1}\right)^{3}+C_{1}\left(\alpha_{1}+i \beta_{1}\right)^{2}+C_{2}\left(\alpha_{1}+i \beta_{1}\right)+C_{3}=0$, comparing real and imaginary parts we get, $\alpha_{1}^{3}-3 \alpha_{1} \beta_{1}^{2}+$ $C_{1}\left(\alpha_{1}^{2}-\beta_{1}^{2}\right)+C_{2} \alpha_{1}+C_{3}=0$,

$3 \alpha_{1}^{2} \beta_{1}-\beta_{1}^{3}+2 C_{1} \alpha_{1} \beta_{1}+C_{2} \beta_{1}=0$

Since $\beta_{1} \neq 0$, eliminating $\beta_{1}$ from the above two equations and then differentiating w.r.to $r$ and putting $\alpha_{1}\left(r_{c}\right)=0$ we obtain $\left.\frac{d \alpha}{d r}\right]_{r=r_{c}}=\operatorname{Real}\left\{\frac{d \lambda_{1,2}}{d r}\right\}_{r=r_{c}}=$ $-\left\{\frac{1}{2\left(C_{2}+C_{1}^{2}\right)} \frac{d\left(C_{1} C_{2}-C_{3}\right)}{d r}\right\}_{r=r_{c}}$.

Since $C_{2}+C_{1}^{2} \neq 0$ the transversality condition reduces to $\left\{\frac{d\left(C_{1} C_{2}-C_{3}\right)}{d r}\right\}_{r=r_{c}} \neq 0$, where

$$
\begin{aligned}
& \left\{\frac{d\left(C_{1} C_{2}-C_{3}\right)}{d r}\right\}_{r=r_{c}}= \\
= & -E\left(C_{1}+\left(C_{2}+d+\gamma\right)(d-r(1-2 S / k))\right. \\
+ & \left.C_{2}(d+\gamma)\right)+F\left(C_{1}+C_{2}(2 d+\mu+\gamma)\right. \\
- & \left.\left(d^{2}+d \gamma+d \mu\right)\right)+\frac{2}{k r}\left(-G+\frac{a_{1} G-2 k a / \beta}{\sqrt{a_{1}^{2}+4 a_{2}}}\right) \\
\cdot\left(C_{1}+C_{2}(2 d+\mu+\gamma-B)-d^{2}\right. & -d \mu-d \gamma+\mu \gamma-B(d+\gamma)) \\
& +(1-2 S / k)\left(C_{1}-C_{2}(2 d+\mu+\gamma-B)\right. \\
& \left.+d^{2}+d \mu+d \gamma+B(d+\gamma)\right),
\end{aligned}
$$


with $G=\frac{k}{2 \beta}\left(\frac{a p}{d+\mu}+b d-a \alpha\right)$,

$$
\begin{aligned}
E & =\frac{p\left(1+2 \alpha S_{1}\right) H}{\left(1+\alpha S_{1}+\beta I_{1}\right)^{2}}-\frac{2 p S_{1}\left(1+\alpha S_{1}\right)}{\left(1+\alpha S_{1}+\beta I_{1}\right)^{3}}(\alpha H+\beta M), \\
F & =\frac{p\left(1+2 \beta I_{1}\right) M}{\left(1+\alpha S_{1}+\beta I_{1}\right)^{2}}-\frac{2 p I_{1}\left(1+\beta I_{1}\right)}{\left(1+\alpha S_{1}+\beta I_{1}\right)^{3}}(\alpha H+\beta M), \\
H & =\frac{1}{r^{2}}\left(-G+\frac{a_{1} G-2 k a / \beta}{\sqrt{a_{1}^{2}+4 a_{2}}}\right) \\
M & =\frac{p-\alpha(d+\mu)}{\beta(d+\gamma)} H
\end{aligned}
$$

\section{NORMAL FORM}

\section{AND CENTER MANIFOLD THEORY}

In this section we shall use the normal form and Center Manifold theorem to study the existence of different bifurcations of the model (1).

Let us shift the origin to any point $\left(S^{*}, I^{*}, R^{*}\right)$ using the transformation $S=S^{\prime}+S^{*}, I=I^{\prime}+I^{*}$ and $R=R^{\prime}+R^{*}$ then the system (1) reduces to the following form (writing omitting the ' sign)

$$
\left\{\begin{array}{l}
\frac{d S}{d t}=a_{11} S+a_{12} I+a_{13} R \\
\quad+a_{14} S^{2}+a_{15} S I+a_{16} I^{2}+O\left(S^{3}, I^{3}\right) \\
\frac{d I}{d t}=a_{21} S+a_{22} I+a_{23} R \\
\quad+a_{24} S^{2}+a_{25} S I+a_{26} I^{2}+O\left(S^{3}, I^{3}\right) \\
\frac{d R}{d t}=a_{31} S+a_{32} I+a_{33} R,
\end{array}\right.
$$

where

$$
\begin{gathered}
a_{11}=r-d-\frac{2 r S^{*}}{k}-\frac{p I^{*}}{\left(1+\alpha S^{*}+\beta I^{*}\right)} \\
+\frac{\alpha p I^{*} S^{*}}{\left(1+\alpha S^{*}+\beta I^{*}\right)^{2}}, \\
a_{12}=-\frac{p S^{*}}{\left(1+\alpha S^{*}+\beta I^{*}\right)}+\frac{p \beta I^{*} S^{*}}{\left(1+\alpha S^{*}+\beta I^{*}\right)^{2}}, \\
a_{13}=\gamma, \\
a_{14}=\frac{\alpha p I^{*}}{\left(1+\alpha S^{*}+\beta I^{*}\right)^{2}}-\frac{p \alpha^{2} I^{*} S^{*}}{\left(1+\alpha S^{*}+\beta I^{*}\right)^{3}}-\frac{r}{k},
\end{gathered}
$$

$$
\begin{aligned}
& a_{15}=-\frac{p)}{\left(1+\alpha S^{*}+\beta I^{*}\right)}+\frac{\alpha p S^{*}+\beta p I^{*}}{\left(1+\alpha S^{*}+\beta I^{*}\right)^{2}} \\
& -\frac{2 \alpha \beta p I^{*} S^{*}}{\left(1+\alpha S^{*}+\beta I^{*}\right)^{3}} \text {, } \\
& a_{16}=\frac{\beta p S^{*}}{\left(1+\alpha S^{*}+\beta I^{*}\right)^{2}}-\frac{p \beta^{2} I^{*} S^{*}}{\left(1+\alpha S^{*}+\beta I^{*}\right)^{3}}, \\
& a_{21}=\frac{p I^{*}}{\left(1+\alpha S^{*}+\beta I^{*}\right)}-\frac{\alpha p I^{*} S^{*}}{\left(1+\alpha S^{*}+\beta I^{*}\right)^{2}}, \\
& a_{22}=\frac{p S^{*}}{\left(1+\alpha S^{*}+\beta I^{*}\right)}-\frac{\beta p S^{*} I^{*}}{\left(1+\alpha S^{*}+\beta I^{*}\right)^{2}}-(d+\mu), \\
& a_{23}=0, a_{24}=-\frac{\alpha p S^{*}}{\left(1+\alpha S^{*}+\beta I^{*}\right)^{2}}+\frac{\alpha^{2} p S^{*} I^{*}}{\left(1+\alpha S^{*}+\beta I^{*}\right)^{3}}, \\
& a_{25}=\frac{p}{\left(1+\alpha S^{*}+\beta I^{*}\right)}-\frac{\alpha p S^{*}+\beta p I^{*}}{\left(1+\alpha S^{*}+\beta I^{*}\right)^{2}} \\
& +\frac{2 \alpha \beta S^{*} I^{*} p}{\left(1+\alpha S^{*}+\beta I^{*}\right)^{3}} \\
& a_{26}-\frac{\beta p S^{*}}{\left(1+\alpha S^{*}+\beta I^{*}\right)^{2}}+\frac{p S^{*} I^{*} \beta^{2}}{\left(1+\alpha S^{*}+\beta I^{*}\right)^{3}}, \\
& a_{31}=0, a_{32}=\mu, a_{33}=-(d+\gamma) .
\end{aligned}
$$

\section{1. Bifurcation analysis of disease free equilibrium $(0,0,0)$}

Now for the equilibrium point $(0,0,0)$ the equation (11) reduces to

$$
\begin{aligned}
\left(\begin{array}{c}
\dot{S} \\
\dot{I} \\
\dot{R}
\end{array}\right) & =\left(\begin{array}{ccc}
r-d & 0 & \gamma \\
0 & -(d+\mu) & 0 \\
0 & 0 & -(d+\gamma)
\end{array}\right)\left(\begin{array}{l}
S \\
I \\
R
\end{array}\right) \\
& +\left(\begin{array}{c}
-\frac{r S^{2}}{k}-p S I \\
p S I \\
0
\end{array}\right)
\end{aligned}
$$

If $r=d$ then one root of the characteristic equation of the system (12) vanishes and other roots (eigenvalues) are $-(d+\mu),-(d+\gamma)$. Then applicability of Theorem 5 fails. In this case we shall investigate the dynamics of (1) near $(0,0,0)$, using center manifold theory $[39,40]$.

The eigenvectors corresponding to the eigenvalues $r-d=$ $0,-(d+\mu)$, and $-(d+\gamma)$ are $\left(\begin{array}{l}1 \\ 0 \\ 0\end{array}\right),\left(\begin{array}{c}-\frac{\gamma}{d+\mu} \\ \frac{\gamma-\mu}{\mu} \\ 1\end{array}\right)$ and 


$$
\begin{gathered}
\left(\begin{array}{c}
-\frac{\gamma}{d+\gamma} \\
0 \\
1
\end{array}\right) \text { respectively. Using the transformation } \\
\qquad\left(\begin{array}{c}
S \\
I \\
R
\end{array}\right)=P\left(\begin{array}{c}
u \\
v \\
w
\end{array}\right)
\end{gathered}
$$

with

$$
P=\left(\begin{array}{ccc}
1 & 0 & 0 \\
-\frac{\gamma}{d+\mu} & \frac{\gamma-\mu}{\mu} & 1 \\
-\frac{{ }_{\gamma}}{d+\gamma} & 0 & 1
\end{array}\right)^{T}
$$

and $\sigma=r-d$ the equation (12) reduces to the following form

$$
\left(\begin{array}{c}
\dot{u} \\
\dot{v} \\
\dot{w}
\end{array}\right)=\left(\begin{array}{ccc}
0 & 0 & 0 \\
0 & -(d+\mu) & 0 \\
0 & 0 & -(d+\gamma)
\end{array}\right)\left(\begin{array}{c}
u \\
v \\
w
\end{array}\right)+\left(\begin{array}{l}
g_{1} \\
g_{2} \\
g_{3}
\end{array}\right),
$$

where $g_{1}=\sigma u+(\sigma+d)\left(a_{1} u^{2}+b_{1} u v+c_{1} v^{2}\right)+d_{1} u v-$ $\frac{\sigma \gamma}{d+\mu} v-\frac{\sigma \gamma}{d+\gamma} w, g_{2}=b_{2} u v+c_{2} w v$ and $g_{3}=-b_{2} u v-$ $c_{2} w v$ with $a_{1}=-\frac{1}{k^{2}}, b_{1}=-\frac{2 c}{k^{2}}-\frac{b\left(d^{2}+d \mu+d \gamma\right)}{d+\mu}, c_{1}=$ $-\frac{c^{2}}{k^{2}}, b_{2}=\frac{p b \mu}{\gamma-\mu}, c_{3}=\frac{p c \mu}{\gamma-\mu}$.

Suppose satisfaction of (12) depends on the bifurcation parameter $\sigma=r-d$. Then considering the bifurcation parameter $\sigma$ as a new independent variable this system (12) can be written in the form

$$
\left(\begin{array}{c}
\dot{u} \\
\dot{v} \\
\dot{w} \\
\dot{\sigma}
\end{array}\right)=\left(\begin{array}{cccc}
0 & 0 & 0 & 0 \\
0 & -(d+\mu) & 0 & 0 \\
0 & 0 & -(d+\gamma) & 0 \\
0 & 0 & 0 & 0
\end{array}\right)\left(\begin{array}{c}
u \\
v \\
w \\
\sigma
\end{array}\right)+\left(\begin{array}{c}
g_{1} \\
g_{2} \\
g_{3} \\
0
\end{array}\right)
$$

Let's assume that there exists a center manifold of the form

$$
\begin{aligned}
& W^{c}(0)=\left\{(u, v, w, \sigma) \in R^{4}: v=h_{1}(u, \sigma),\right. \\
& w=h_{2}(u, \sigma),|u|<\delta,|\sigma|<\delta_{1}, h_{i}(0,0)=0 \\
& \left.D h_{i}(0,0)=0, i=1,2\right\},
\end{aligned}
$$

where $\delta>0$ and $\delta_{1}>0$ sufficiently small and $D$ denotes derivative with respect to $u$.

Now we choose

$$
\left\{\begin{array}{l}
v=h_{1}(u, \sigma)=a_{11} u^{2}+a_{12} u \sigma+a_{13} \sigma^{2}+\ldots \\
w=h_{2}(u, \sigma)=a_{21} u^{2}+a_{22} u \sigma+a_{23} \sigma^{2}+\ldots
\end{array}\right.
$$

along with $h_{1}$ and $h_{2}$ satisfying the equation [35]

$$
D\left(\begin{array}{l}
h_{1} \\
h_{2}
\end{array}\right) g_{1}-B\left(\begin{array}{l}
h_{1} \\
h_{2}
\end{array}\right)=\left(\begin{array}{l}
g_{2} \\
g_{3}
\end{array}\right)
$$

and the condition $h(0)=D h(0)=0$.

Then substituting (15) in (16) and comparing the coefficients of similar power of $u^{2}, u \sigma$ and $\sigma^{2}$ on both sides of the equation we get

$$
\begin{aligned}
& u^{2}:(d+\mu) a_{11}=a_{1} d,(d+\gamma) a_{21}= \\
= & a_{1} d \Longrightarrow a_{11}=\frac{d a_{1}}{d+\mu}, \\
& a_{21}=\frac{d a_{1}}{d+\gamma} \\
u \sigma: & a_{12}=0, a_{23}=0 \\
\sigma^{2}: & a_{13}=a_{23}=0
\end{aligned}
$$

Hence from equation (14) we get

$$
\begin{aligned}
& \dot{u}=u \sigma+d a_{1} u^{2}+\left(a_{1} \sigma-a_{11} \frac{\sigma \gamma}{d+\mu}\right) u^{2} \\
& +o\left(\sigma, u^{3}\right)=f(u . \sigma)(\text { say }), \\
& \quad \dot{\sigma}=0 .
\end{aligned}
$$

Here $f(0, \sigma)=0, \frac{\partial f(0,0)}{\partial u}=0, \frac{\partial^{2} f(0,0)}{\partial u \partial \sigma} \frac{\partial^{2} f(0,0)}{\partial^{2} u}=$ $2 d a_{1} \neq 0$

Hence the system will go through transcritical bifurcation at $(0,0,0)$, [37]. From the above discussion we can summarize the following theorem:

Theorem 12. If $r=d$ then solution of the system (1) in the neighbourhood of the trivial equilibrium point goes through transcritical bifurcation.

\section{2. Bifurcation analysis of disease free equilibrium} $\left(k\left(1-\frac{d}{r}\right), 0,0\right)$

For the disease free equilibrium point $\left(k\left(1-\frac{d}{r}\right), 0,0\right)$ the system (11) reduces to (using the same symbols as in (11))

$$
\begin{aligned}
\left(\begin{array}{c}
\dot{S} \\
\dot{I} \\
\dot{R}
\end{array}\right) & =\left(\begin{array}{lll}
a_{11} & a_{12} & a_{13} \\
a_{21} & a_{22} & a_{23} \\
a_{31} & a_{32} & a_{33}
\end{array}\right)\left(\begin{array}{c}
S \\
I \\
R
\end{array}\right) \\
& +\left(\begin{array}{c}
a_{14} S^{2}+a_{15} S I+a_{16} I^{2} \\
a_{24} S^{2}+a_{25} S I+a_{26} I^{2} \\
0
\end{array}\right)
\end{aligned}
$$

where

$a_{11}=-(r-d)$, 


$$
\begin{aligned}
a_{12}=-\frac{p k\left(1-\frac{d}{r}\right)}{1+\alpha k\left(1-\frac{d}{r}\right)}, \\
a_{13}=\gamma, a_{21}=0, \\
a_{22}=(d+\mu)\left(R_{10}-1\right), \\
a_{23}=0, a_{31}=0, \\
a_{32}=\mu, a_{33}=-(d+\gamma), a_{14}=-\frac{r}{k} \\
a_{15}=-\frac{p}{1+\alpha k\left(1-\frac{d}{r}\right)}, \\
a_{24}=-\frac{\alpha S_{0} p}{\left(1+\alpha S_{0}\right)^{2}}, \\
a_{25}=-\frac{p}{\left(1+\alpha S_{0}\right)^{2}}, \\
a_{26}=-\frac{\beta S_{0} p}{\left(1+\alpha S_{0}\right)^{2}} .
\end{aligned}
$$

The eigenvalues of the corresponding linear system are $\lambda_{1}=a_{11}, \lambda_{2}=a_{22}$ and $\lambda_{3}=a_{33}$. If the basic reproduction number $R_{0}=1$, the equilibrium point $\left(S_{0}, 0,0\right)$ is of nonhyperbolic type then the usual eigen analysis fails to study the behavior of the equilibrium point. Then it is necessary to take help of the center manifold theorem. Using the transformation

$$
\left(\begin{array}{l}
S \\
I \\
R
\end{array}\right)=Q\left(\begin{array}{c}
u \\
v \\
w
\end{array}\right)
$$

the system of equation (17) reduces to the form

$$
\left(\begin{array}{c}
\dot{u} \\
\dot{v} \\
\dot{w}
\end{array}\right)=\left(\begin{array}{ccc}
a_{11} & 0 & 0 \\
0 & 0 & 0 \\
0 & 0 & a_{33}
\end{array}\right)\left(\begin{array}{c}
u \\
v \\
w
\end{array}\right)+\left(\begin{array}{l}
g_{11} \\
g_{22} \\
g_{33}
\end{array}\right)
$$

where:

$Q=\left(\begin{array}{lll}1 & 0 & 0 \\ a & c & 1 \\ b & 0 & 1\end{array}\right)^{T}$

$a=\frac{a_{12}(d+\gamma+\mu \gamma)}{\mu(r-d)}$,

$b=\frac{\gamma}{r-2 d-\gamma}$

$c=\frac{d+\gamma}{\mu}$,

$g_{11}=A_{11} u^{2}+B_{11} v^{2}+C_{11} w^{2}+D_{11} u v+E_{11} u w+F_{11} v w$, $g_{22}=A_{22} u^{2}+B_{22} v^{2}+C_{22} w^{2}+D_{22} u v+E_{22} u w+F_{22} v w$, $g_{33}=A_{33} u^{2}+B_{33} v^{2}+C_{33} w^{2}+D_{33} u v+E_{33} u w+F_{33} v w$, with,

$$
\begin{aligned}
& A_{11}=a_{14}-a_{24} \frac{a-b}{c}, \\
& B_{11}=\left(a_{14}-a_{24} \frac{a-b}{c}\right) a^{2}+\left(a_{15}-a_{25} \frac{a-b}{c}\right) a c+ \\
& \left(a_{16}-a_{26} \frac{a-b}{c}\right) c^{2}, C_{11}=\left(a_{14}-a_{24} \frac{a-b}{c}\right) b^{2},
\end{aligned}
$$

$$
\begin{aligned}
& D_{11}=2 a\left(a_{14}-a_{24} \frac{a-b}{c}\right)+c\left(a_{14}-a_{24} \frac{a-b}{c}\right), \\
& E_{11}=2 b\left(a_{14}-a_{24} \frac{a-b}{c}\right), \\
& F_{11}=2 a b\left(a_{14}-a_{24} \frac{a-b}{c}\right)+b c\left(a_{15}-a_{25} \frac{a-b}{c}\right), \\
& A_{22}=-A_{33}=\frac{a_{24}}{c}, \\
& B_{22}=-B_{33}=\frac{a^{2} a_{24}+a c a_{25}+a_{26} c^{2}}{c} \\
& C_{22}=-C_{33}=\frac{a_{24} b^{2}}{c}, \\
& D_{22}=-D_{33}=\frac{2 a a_{24}+c a_{25}}{c}, \\
& E_{22}=-E_{33}=\frac{2 b a_{24}}{c}, \\
& F_{22}=-F_{33}=\frac{2 a b a_{24}+b c a_{25}}{c} .
\end{aligned}
$$

The system (18) can be written in the following form

$$
\left\{\begin{array}{l}
\dot{v}=g_{22} \\
\dot{u}=a_{11} u+g_{11} \\
\dot{w}=a_{33} w+g_{33}
\end{array}\right.
$$

By the center manifold theorem [37] there exists a center manifold of the system (19) which can be expressed by

$$
\begin{aligned}
& W^{c}(0)=\left\{(u, v, w) \in R^{3}: u=h_{1}(v),\right. \\
& \left.w=h_{2}(v),|u|<\delta, h_{i}(0)=0, D h_{i}(0)=0, i=1,2\right\},
\end{aligned}
$$

$\delta$ is sufficiently small, $D h$ is the derivative of $h$ with respect to $v$. To compute the center manifold $W^{c}(0)$, we assume

$$
\begin{gathered}
u=h_{1}(v)=h_{11} v^{2}+h_{12} v^{3}+\ldots \\
w=h_{2}(v)=h_{12} v^{2}+h_{22} v^{3}+\ldots,
\end{gathered}
$$

which satisfies the equation

$$
D\left(\begin{array}{l}
h_{1} \\
h_{2}
\end{array}\right) g_{22}-B\left(\begin{array}{l}
h_{1} \\
h_{2}
\end{array}\right)=\left(\begin{array}{l}
g_{11} \\
g_{33}
\end{array}\right) .
$$

Substituting $h_{1}$ and $h_{2}$ in equation (20) and comparing the like powers of $v$ we get $h_{11}=\frac{B_{11}}{r-d}$,

$$
\begin{aligned}
& h_{21}=\frac{B_{33}}{d+\gamma}, \\
& h_{12}=\frac{D_{11} h_{11}+F_{11} h_{21}-2 h_{11} B_{22}}{r-d}, \\
& h_{22}=\frac{D_{33} h_{11}+f_{33} h_{21}-2 h_{21} B_{22}}{d+\gamma} .
\end{aligned}
$$


$\therefore$ Putting the expression of $u$ and $w$ in the first equation of (19) we get

$$
\dot{v}=B_{22} v^{2}+v^{3}\left\{D_{22} h_{11}+F_{22} h_{21}+o\left(v^{4}\right)\right\}
$$

Hence this equilibrium point is a saddle-node type. Therefore, the reduced system depicted by (17) shows that this system is unstable and consequently the original system is unstable. Hence from the above discussion we can summarize the following theorem:

Theorem 13. If $R_{0}=1$ then solution of the system (1) in the neighbourhood of the disease free equilibrium is saddle-node type and consequently unstable.

\section{3. Bifurcation analysis of endemic equilibrium}

For the endemic equilibrium point $\left(S_{1}, I_{1}, R_{1}\right)$ the system (11) reduces (using the same same the symbols as in (11)) to

$$
\begin{aligned}
\left(\begin{array}{c}
\dot{S} \\
\dot{I} \\
\dot{R}
\end{array}\right) & =\left(\begin{array}{ccc}
a_{11} & a_{12} & a_{13} \\
a_{21} & a_{22} & 0 \\
0 & a_{32} & a_{33}
\end{array}\right)\left(\begin{array}{c}
S \\
I \\
R
\end{array}\right) \\
& +\left(\begin{array}{c}
a_{14} S^{2}+a_{15} S I+a_{16} I^{2} \\
a_{24} S^{2}+a_{25} S I+a_{26} I^{2} \\
0
\end{array}\right)
\end{aligned}
$$

where all $a_{i j}$ are the same as coefficients in (11), only $\left(S^{*}, I^{*}, R^{*}\right)$ will be replaced by $\left(S_{1}, I_{1}, R_{1}\right)$. Equation (10) represents the characteristics equation of the linear part of it. If it satisfies the conditions of Theorem 11 the roots of the equation (10) have one negative real root and a pair of purely imaginary roots and the system undergoes Hopf bifurcation. To verify the stability of Hopf bifurcation in the neighbourhood of endemic equilibrium point let us choose the transformation

$$
\left(\begin{array}{c}
S \\
I \\
R
\end{array}\right)=P\left(\begin{array}{c}
u \\
v \\
w
\end{array}\right)
$$

where: $P=\left(\begin{array}{ccc}p_{11} & p_{12} & p_{13} \\ p_{21} & p_{22} & p_{23} \\ p_{3} & 0 & p_{33}\end{array}\right), p_{11}=-\frac{a_{33}}{a_{32}}$,

$p_{12}=-\frac{\sqrt{C_{2}}}{a_{32}}, p_{13}=-\frac{a_{33}+C_{1}}{a_{32}}, p_{21}=-\frac{a_{22}}{a_{21}}$,

$p_{22}=-\frac{\sqrt{C_{2}}}{a_{21}}, p_{23}=-\frac{a_{22}+C_{1}}{a_{21}}, p_{31}=1, p_{33}=1$.

Let $P^{-1}=\left(\begin{array}{lll}s_{11} & s_{12} & s_{13} \\ s_{21} & s_{22} & s_{23} \\ s_{31} & S_{32} & s_{33}\end{array}\right)$,

$s_{11}=\frac{p_{33} p_{22}}{|P|}$,

$s_{12}=-\frac{p_{33} p_{12}}{|P|}$, $s_{13}=\frac{p_{12} p_{23}-p_{13} p_{22}}{|P|}$,

$s_{21}=-\frac{p_{21} p_{33}-p_{23} p_{31}}{|P|}$,

$s_{22}=\frac{p_{11} p_{33}-p_{13} p_{31}}{|P|}$,

$s_{23}=-\frac{p_{11} p_{23}-p_{13} p_{21}}{|P|}$

$s_{31}=-\frac{p_{22} p_{31}}{|P|}, s_{32}=\frac{p_{12} p_{31}}{|P|} s_{33}=-\frac{p_{22} p_{11}-p_{12} p_{21}}{|P|}$,

where $|P|$ denotes the determinant value of $P$.

Using the above transformation, the system (21) reduces to the form

$$
\left(\begin{array}{c}
\dot{u} \\
\dot{v} \\
\dot{w}
\end{array}\right)=\left(\begin{array}{ccc}
0 & -\sqrt{C_{2}} & 0 \\
\sqrt{C_{2}} & 0 & 0 \\
0 & 0 & -C_{1}
\end{array}\right)\left(\begin{array}{c}
u \\
v \\
w
\end{array}\right)+\left(\begin{array}{l}
f_{1} \\
f_{2} \\
f_{3}
\end{array}\right),
$$

where:

$f_{1}=B_{11} u^{2}+B_{12} v^{2}+B_{13} w^{2}+B_{14} u v+B_{15} u w+B_{16} v w$,

$f_{2}=B_{21} u^{2}+B_{22} v^{2}+B_{23} w^{2}+B_{24} u v+B_{25} u w+B_{26} v w$,

$f_{3}=B_{31} u^{2}+B_{32} v^{2}+B_{33} w^{2}+B_{34} u v+B_{35} u w+B_{36} v w$, with,

$$
\begin{aligned}
& B_{11}=P_{11}^{2} A_{11}+A_{13} P_{21}^{2}+A_{12} P_{11} P_{21}, \\
& B_{12}=P_{12}^{2} A_{11}+A_{13} P_{22}^{2}+A_{12} P_{12} P_{22}, \\
& B_{13}=P_{13}^{2} A_{11}+A_{13} P_{23}^{2}+A_{12} P_{13} P_{23},
\end{aligned}
$$

$B_{14}=2 A_{11} P_{11} P_{12}+2 A_{13} P_{21} P_{22}+A_{12}\left(P_{11} P_{22}+P_{12} P_{21}\right)$,

$B_{15}=2 A_{11} P_{11} P_{13}+2 A_{13} P_{21} P_{23}+A_{12}\left(P_{11} P_{23}+P_{21} P_{13}\right)$,

$B_{16}=2 A_{11} P_{12} P_{13}+2 A_{13} P_{22} P_{23}+A_{12}\left(P_{12} P_{23}+P_{13} P_{22}\right)$.

Similar expression for $B_{2 i}$ and $B_{3 i}$ is obtained by replacing $A_{1 j}$ by $A_{2 j}$ and $A_{3 j}$, respectively. Where

$$
\begin{aligned}
& A_{11}=s_{11} a_{14}+s_{12} a_{24}, \\
& A_{12}=s_{11} a_{15}+s_{12} a_{25}, \\
& A_{13}=s_{11} a_{16}+s_{12} a_{26}, \\
& A_{21}=s_{21} a_{14}+s_{22} a_{24}, \\
& A_{22}=s_{21} a_{15}+s_{22} a_{25}, \\
& A_{23}=s_{21} a_{16}+s_{22} a_{26}, \\
& A_{31}=s_{31} a_{14}+s_{32} a_{24}, \\
& A_{32}=s_{31} a_{15}+s_{32} a_{25}, \\
& A_{33}=s_{31} a_{16}+s_{32} a_{26} .
\end{aligned}
$$

There exists a center manifold for (22) which can be represented as follows

$$
\begin{aligned}
& W^{c}(0)=\left\{(u, v, w) \in R^{3}: w=h(u, v),\right. \\
& |w|<\delta, h(0,0)=0, \operatorname{Dh}(0,0)=0\},
\end{aligned}
$$


where $\delta$ is sufficiently small, $D h$ is the partial derivative of $h$ with respect to $u, v$.

To compute the center manifold $W^{c}(0)$, we assume that $w=h(u, v)=b_{1} u^{2}+b_{2} v^{2}+b_{3} u v+o\left(|u|^{4}\right)$.

In case of disease free and trivial equilibrium, using the conditions satisfied by $h(u, v)$, we obtain

$$
\begin{gathered}
b_{1}=\frac{B_{31}-\sqrt{C_{2}}}{C_{1}}, \\
b_{3}=\frac{B_{34}+2 b_{1} \sqrt{c_{2}}}{\sqrt{c_{2}}+C_{1}}
\end{gathered}
$$

and

$$
\begin{array}{cc}
b_{2}=\frac{B_{32}+b_{3} \sqrt{c_{2}}}{C_{1}} . & \begin{array}{l}
\text { instability of the periodic orbit about the endemic equilibrium } \\
\text { point, [40]. }
\end{array} \\
\left.\left.\sigma=\frac{1}{16}\left\{f_{u u u}+f_{u v v}+g_{u u v}+g_{v v v}\right\}+\frac{1}{16 \sqrt{C_{2}}}\left\{f_{u v}\left(f_{u u}+f_{v v}\right)-g_{u v}\left(g_{u u}+g_{v v}\right)-f_{u u}\right) g_{u u}+f_{v v}\right) g_{v v}\right\} \\
=\frac{1}{16}\left\{6 B_{15} b_{1}+2\left(B_{15} b_{2}+B_{16} b_{3}\right)+2\left(B_{16} b_{3}+B_{26} b_{1}\right)+6 B_{26} b_{2}\right\} \\
+\frac{1}{16 \sqrt{C_{2}}}\left\{2 B_{14}\left(B_{11}+B_{12}\right)-2 B_{24}\left(B_{21}+B_{22}\right)-B_{11} B_{21}+B_{12} B_{22}\right\} .
\end{array}
$$

Now we compute $\sigma$ - defined below, it gives the stability or
From the above discussion we can summarize the following theorem:

Theorem 14. The periodic solution that is emanating from the endemic equilibrium point $\left(S_{1}, I_{1}, R_{1}\right)$ in the neighbourhood of $r_{c}$ is stable (unstable) if $\sigma<0(>0)$. Moreover, the system is said to be super critical (sub critical) if $\sigma<0(>0)$.

\section{NUMERICAL SIMULATION}

To verify the theoretical findings numerical simulation is performed considering empirical values of the parameters. We consider first $k=100, p=0.010, \beta=0.01, d=$ $2.3, \gamma=0.001, \mu=0.01, \alpha=0.021, r=0.73$ then only the trivial equilibrium point $(0,0,0)$ exists because $r<d$. By Theorem 5 this equilibrium point is locally asymptotically stable. Again the conditions of global stability of Theorem 6 are satisfied hence the trivial equilibrium point $(0,0,0)$ is globally asymptotically stable. The stability and global stability is justified from Fig. 1(a) and Fig. 1(b), respectively. From Fig. 1(b) it is clear that from different starting points the solution ultimately converges to the equilibrium point $(0,0,0)$.

For existence of disease free equilibrium points we have $r>d$, so increase the value of $r$ keeping all other parameters fixed as in the previous one. Consider $r=8.73$ then the trivial equilibrium point is unstable (by Theorem 6) and consequently the disease free equilibrium point $(73.6541,0,0)$ exists. For this parameter values we have $R_{0}=0.1252<1$, $R_{01}=0.1530<1$, hence the endemic equilibrium point does not exists and the disease free equilibrium is locally asymptotically stable in nature. Fig. 2 (a)-(b) shows the locally asymptotic stable and global asymptotic stable behavior of the disease free equilibrium point. In Fig. 2(b) the solutions start from different initial points and ultimately go to the disease free equilibrium point.

For existence of the endemic equilibrium point, the rate of infection should be higher. Thus we increase the value of $p, r$. Consider $p=3.010, r=3.73$ keeping the remaining parameters fixed we obtained the endemic equilibrium point $(0.7837,0.4752,0.0021)$ (all values are taken as correct up to four decimal place). Here $R_{0}=27.6746>1, R_{01}=$ $1.0047>1$ and the conditions of Theorem 9 are satisfied. Hence this equilibrium point is stable in nature. The conditions of Theorem 10 are also satisfied for the considered values of the parameters. The corresponding numerical solution of the differential equations is shown in Fig. 3(a-b). From Fig. 3 it is clear that the system (1) is globally asymptotically stable in the neighbourhood of this endemic equilibrium point. 
To study the Hopf bifurcation we consider $\alpha=0.47$ 9980, $r=r_{c}=2.407734$ and keeping other parameters fixed, we obtain the endemic equilibrium point as $(1.1605,0.0400,0.0001)$. Then roots of the equation (10) are: $-2.3010,-0.0000 \pm 0.3435 i$. Since one root is negative and the other two have zero real part, the solution in the neighbourhood of the equilibrium point will be periodic and the endemic equilibrium point is a center. The value of

$$
\left[\frac{d(\operatorname{Real} \lambda)}{d r}\right]_{r=r_{c}} \neq 0
$$

hence the system will go through Hopf bifurcations. Since for $r=2.36<r_{c}$ the system is stable and trajectory is a stable spiral which is shown in Fig. 4(a). The bifurcation occurs at $r=r_{c}$ and for $r>r_{c}$ the stable spiral jumps to an unstable spiral, finally closed orbit (limit cycle) arises after the bifurcation, which is justified by figure $4(b)$.

Next we draw the dynamical behavior of the solution of the system (1) through bifurcation study of different parameters $r, \alpha$ and $\beta$. Fig. 5 shows the bifurcation diagram of the system (1) with respect to intrinsic growth rate $r$ within the interval $0<r \leq 10$. From the figure it is clear that all the populations remain in the stable state in the range $0<r<2.407734$ and then the system has limit cycle oscillation for all the populations. From this analysis it is clear that if the rate of infection is high (here $p=3.010$ ) then the population density oscillates for high intrinsic growth rate $(r>2.407734)$ and elimination of the disease is impossible. Again from Fig. 5 it is clear that bifurcation is super-critical with respect to the intrinsic growth rate. Biologically it is most important if both: the rate of infection and the growth rate is high, then controlling the disease is quite difficult.

Fig. 6 represents the bifurcation diagram with respect to the inhibition coefficient $(\alpha)$ in the range $0<\alpha \leq 4$.

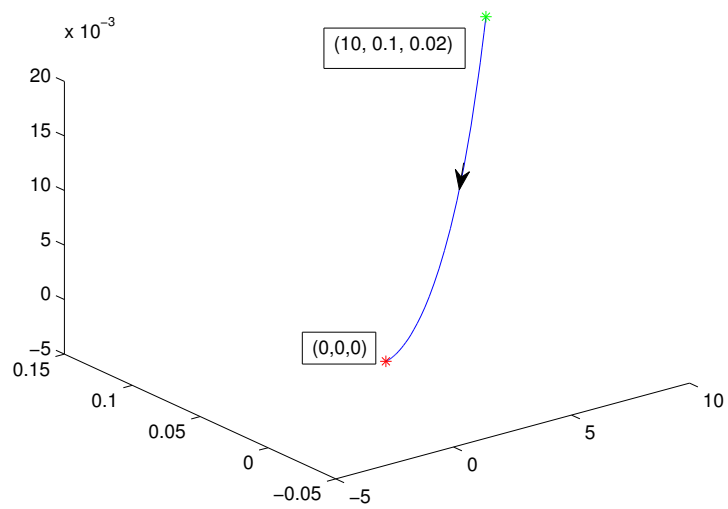

(a)
From the figure it is clear that for $0<\alpha \leq 0.47998$ the number of all populations will increase, then in the range $0.47998<\alpha \leq 1.28$ the susceptible populations becomes periodic and other two population start to decrease and ultimately when $\alpha \geq 1.28$ the number of infected and recovered populations diminish and the susceptible populations becomes stable in nature. Thus the inhibition parameter $(\alpha)$ may be taken as an controlling parameter for controlling the infection. Biologically it is the most important because if we increase the social awareness programme then many diseases are easy to control.

Fig. 7(a,b) represents the bifurcation diagram with respect to the another inhibition coefficient $(\beta)$ here considered as the crowding effect or protection measure in the range $0<\beta \leq 1$. From Fig. 7(a) it is clear that the all populations have limit cycle oscillations in the range $0<\beta \leq 0.31$. All the population settle down to their respective steady state. With the increase of $\beta$ the infected and recovered class diminishes and the susceptible class become stable, which is clear from Fig. 7(b).

\section{CONCLUSIONS}

In our considered model there are three equilibrium points. One of them is the trivial equilibrium point, second is the disease free equilibrium point and the third is the endemic equilibrium point. The trivial equilibrium point always exists, the disease free equilibrium point exists if $r>d$ and the endemic equilibrium point exists if $R_{01}>1$. The disease free equilibrium point is globally asymptotically stable under certain conditions. The endemic equilibrium point is globally asymptotically stable in the region $\Omega_{1}=\left\{(S, I, R): 1<\frac{I_{1}}{I}<\frac{R_{1}}{R}<\frac{S_{1}}{S}\right\}$, when $R_{01}>1$

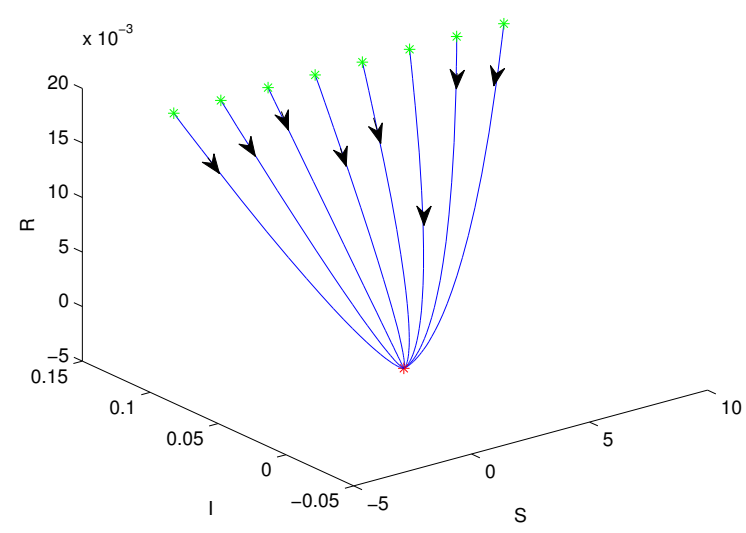

(b)

Fig. 1. Phase portrait in the S-I-R space for $k=100, p=0.010, \beta=0.01, d=2.3, \gamma=0.001, \mu=0.01, \alpha=0.021, r=0.73$ 


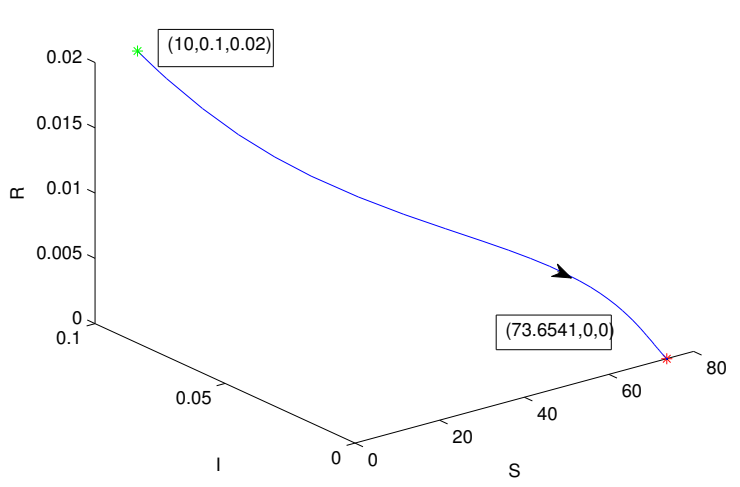

(a)



(b)

Fig. 2. Phase portrait in the S-I-R space for $k=100, p=0.010, \beta=0.01, d=2.3, \gamma=0.001, \mu=0.01, \alpha=0.021, r=8.73$

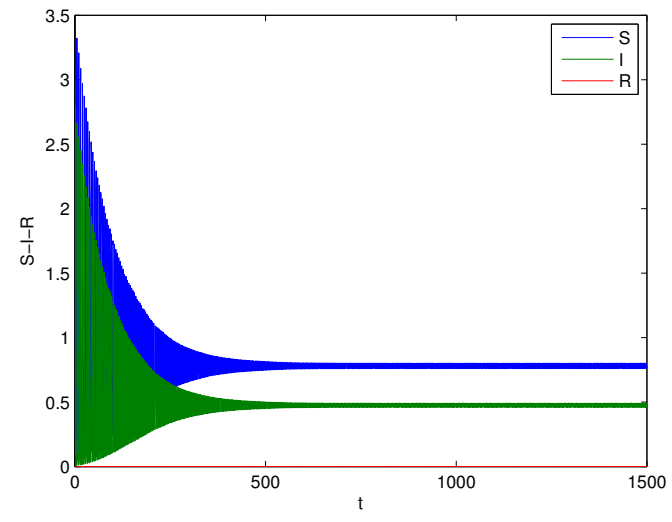

(a)

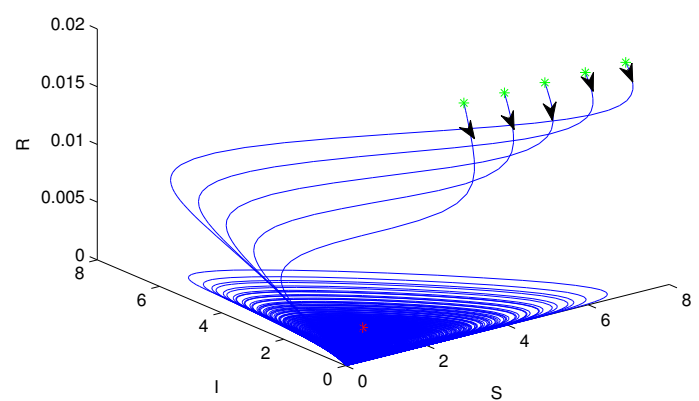

(b)

Fig. 3. (a) Time series of S-I-R plot for $k=100, p=3.010, \beta=0.01, d=2.3, \gamma=0.001, \mu=0.01, \alpha=0.021, r=3.73$. (b) The phase portrait for the global stability for the same values of the parameters

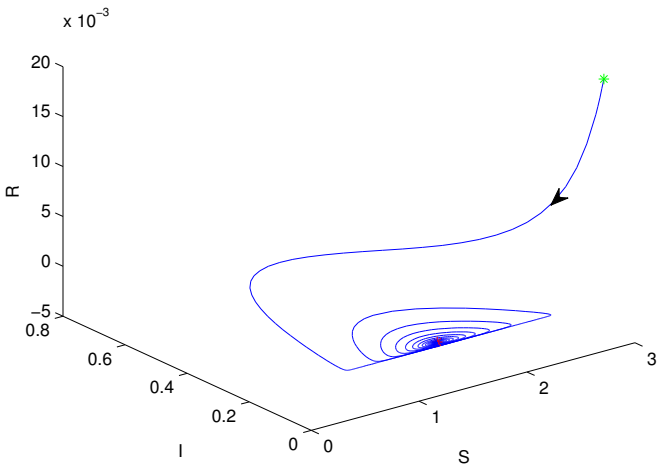

(a)

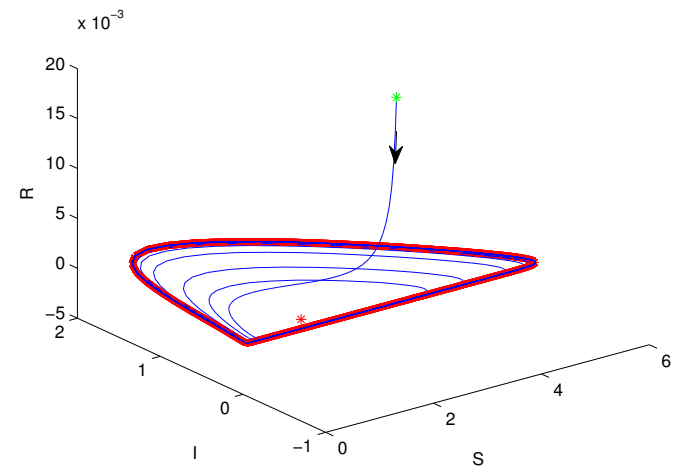

(b)

Fig. 4. Phase portrait for $k=100, p=3.010, \beta=0.01, d=2.3, \gamma=0.001, \mu=0.01, \alpha=0.479980$ (a) for $r<r_{c}$, (b) for $r>r_{c}$, the bold red line corresponds to the stable limit cycle 

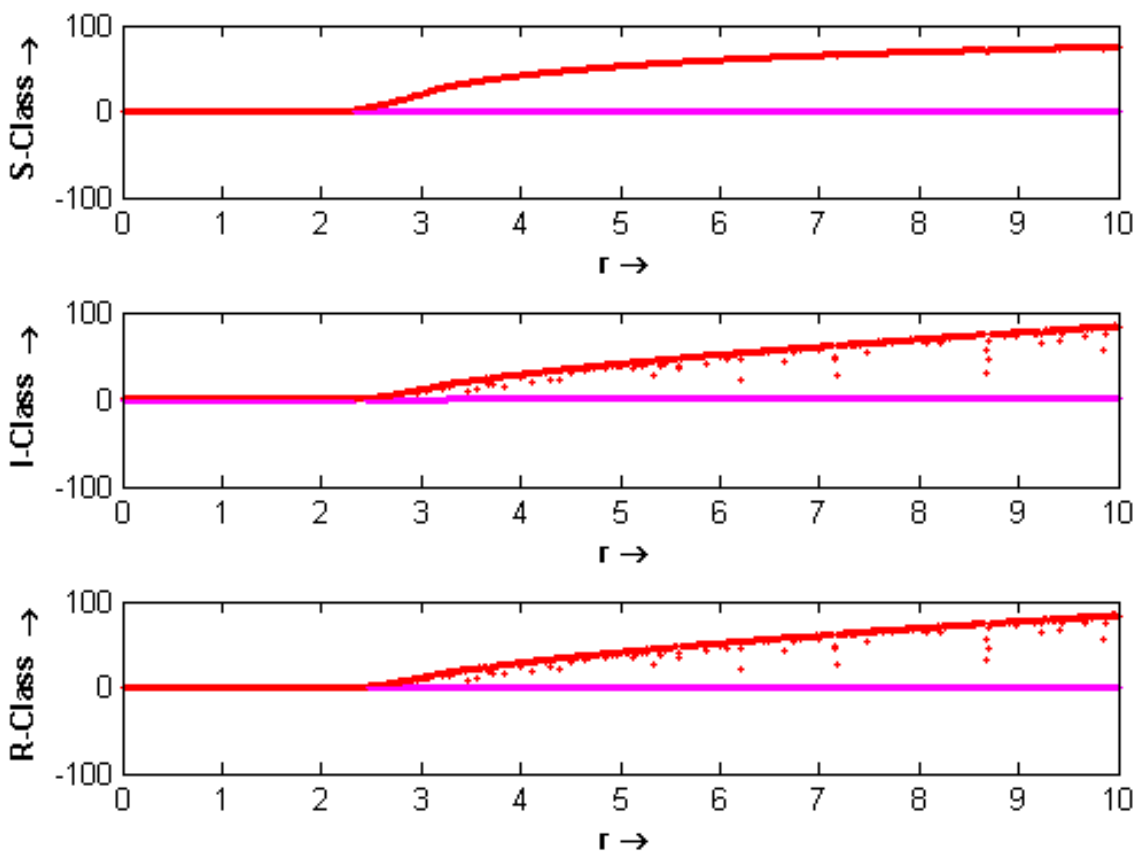

Fig. 5. The bifurcation diagram with respect to the intrinsic growth rate $(r)$ and the other parameters are $k=100, p=3.010, \beta=0.01$, $d=2.3, \gamma=0.001, \mu=0.01, \alpha=0.479980$
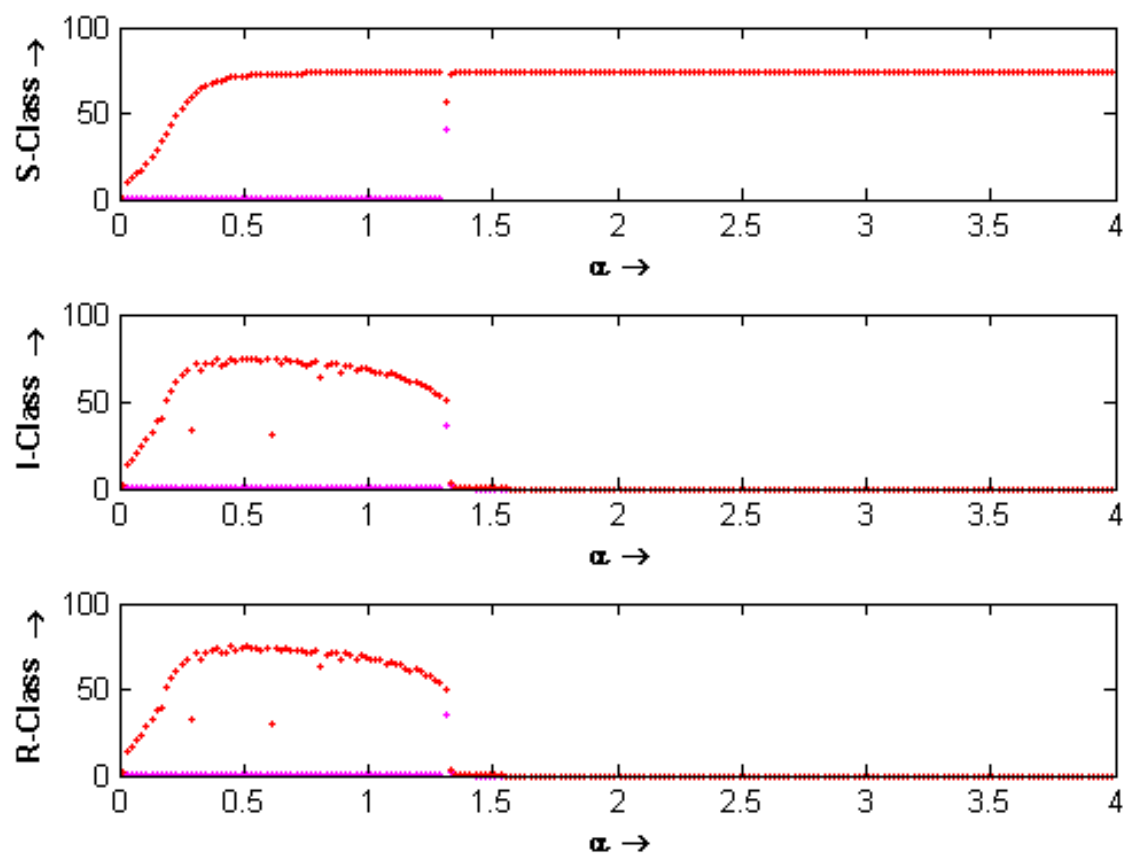

Fig. 6. The bifurcation diagram with respect to the inhibition coefficient $(\alpha)$ and the other parameters are: $k=100, p=3.010$, $\beta=0.01, d=2.3, \gamma=0.001, \mu=0.01$, and $r=8.702$ 



(a)


(b)

Fig. 7. The bifurcation diagram with respect to the inhibition coefficient $(\beta)$ and the other parameters are $k=100, p=3.010$, $d=2.3, \gamma=0.001, \mu=0.01, \alpha=0.47998$ and $r=8.702$ (a) For $0<\beta \leq 10$ and (b) Local amplification of Fig. (a) in $0<\beta \leq 1$

and goes through super-critical Hopf bifurcation depending on the intrinsic growth rate. From the analysis of the bifurcation diagrams (Fig. 5-7) it is clear that the intrinsic growth rate $(r)$ and the inhibition coefficients ( $\alpha$ and $\beta$ ) play an important role in controlling the disease. If the rate of infection is low then at high intrinsic growth rate the disease is easy to control. But if the rate of infection is high then at high intrinsic growth rate the system shows oscillatory behaviour and consequently eradication of the disease is impossible. Again from the bifurcation diagram (Fig. 6, 7) it is clear that if the inhibition coefficients are high, then eradication of the disease is easy. So to control the disease, the social awareness program and the protection measure play an important role.

\section{Acknowledgement}

The authors are grateful to the anonymous referee for careful checking of the details and for helpful comments that improved this paper. 


\section{References}

[1] Z. Hu, Z. Teng, L. Zhang, Stability and bifurcation analysis in a discrete SIR epidemic model, Mathematics and computers in Simulation 97, 80-93 (2014).

[2] W.M. Liu, S.A. Levin, Y. Iwasa, Influence of nonlinear incidence rates upon the behavior of SIRS epidemiological models, J. Math. Biol 23, 187-204 (1986).

[3] H.W. Hethcote, M.A. Lewis, P. van den Driessche, An epidemiological model with delay and a nonlinear incidence rate, J. Math. Biol. 27, 49-64, (1989).

[4] D. Xiao, S. Ruan, Global analysis of an epidemic model with nonmonotone incidence rate, Math. Biosci. 208, 419-429 (2007).

[5] H.N. Agiza, E.M. Elabbasy, EL-Metwally, A.A. Elsadany, Chaotic dynamics of a discrete prey-predator model with Holling type-II, Non-linear Annal. Real World Appl. 10, 116129 (2009).

[6] W.O. Kermack, A.G. McKendrick, Contribution to mathematical theory of epidemics, P. Roy. Soc. Lond. A Mat. 115, 700-721 (1927).

[7] N.T.J. Bailey, The Mathematical Theory of Infectious Diseases, Griffin, London, 1975.

[8] J.D. Murray, Mathematical Biology, Springer, New York, 1993.

[9] Z. Ma, J. Li (eds.), Dynamical Modeling and Analysis of Epidemics, World Scientific, 2009.

[10] R.M. Anderson, R.M. May, Infectious Diseases of Humans: Dynamics and Control. Oxford University Press, 1998.

[11] F. Brauer, C. Castillo-Chavez, Mathematical Models in Population Biology and Epidemiology, Springer, 2001.

[12] M.Y. Li, L. Wang, Global stability in some SEIR epidemic models, IMA Volumes in Mathematics and its Application, Spring-Verlag 126, 295-311 (2002).

[13] Q. Zou, S. Gao, Q. Zhong, pulse vaccination strategy in an epidemic model with time delay and nonlinear, Advanced Studies in Biology 7(1), 307-321 (2009).

[14] T. Zhang, Z. Teng, Global asymptotic stability of a delayed SEIRS epidemic model with saturation incidence, Chaos Soliton and Fractals 37, 1450-1468 (2008).

[15] L. Zhou, M. Fan, Dynamics of an SIR epidemic model with limited medical resources revisited, Nonlinear Anal RWA 13, 312-324 (2012).

[16] F. Brauer, P. van den Driessche, Models for transmission of disease with immigration of infectives, Math Biosci 171(2), 143-154 (2001).

[17] U. Ghosh, S. Sarkar, D.K. Khan, Modelling of infectious disease in Presence of Vaccination and Delay, International Journal of Epidemiology and Infection 2(3), 50-57 (2014).

[18] Y. Xue, T. Li, Stability and Hopf Bifurcation for a Delayed SIR Epidemic Model with Logistic Growth, Hindawi Publishing Corporation 1-11 (2013).

[19] Y. Enatsu, E. Messina, Y. Muroya, Y. Nakata, E. Russo, A. Vecchio, Stability analysis of delayed SIR epidemic model with a class of nonlinear incidence rates, Applied Mathematics and Computation 218, 5327-5336 (2012).

[20] R. Ghosh, U. Ghosh, Bifurcation analysis of SIR Model with Logistically Grown Susceptibles and Effect of Loss of Immunity of the Recovered Class, Proceeding: International seminar: Recent trends in Mathematics, Calcutta University, Applied Mathematics, Springer Proceedings 227-235 (2015).
[21] A. Kaddar, Stability analysis of delayed SIR epidemic model with saturated incidence rate, Nonlinear Analysis 3, 299-306 (2010).

[22] A. Karobeinikov, P.K. Maini, Nonlinear incidence and stability of infectious disease models, Math. Med. Bil. 22, 113-128 (2005).

[23] B. Dubey, A. Parta, P.K. Srivastava, U.S. Dubey, Modeling and analysis of an SEIR model with different type of nonlinear treatment rates, Journal of Biological System 2(3), 1-25 (2013).

[24] B. Dubey, P. Dubey, U.S. Dubey, Dynamics of an SIR epidemic model with non-linear incidence rate and treatment rate, Applications and Applied Mathematics 10(2), 718-737 (2015).

[25] T.K. Kar, P.K. Mondal, Global dynamics and bifurcation in delayed SIR epidemic model, Nonlinear Analysis: Real World Applications 55, 710-722 (2011).

[26] M. Agarwal, V. Verma, Modeling and Analysis of the Spread of an Infectious Disease Cholera with Environmental Fluctuations, Applications and Applied Mathematics 7(1), 406-425 (2012)

[27] P.K. Mondal, T.K. Kar, Global Dynamics of a Water-Borne Disease Model with Multiple Transmission Pathways, Applications and Applied Mathematics 8(1), 99-115 (2013).

[28] C. Wei, L. Chen, A Delayed Epidemic Model with Pulse Vaccination, Discrete Dynamics in Nature and Society (2008).

[29] J.Z. Zhang, Z. Jin, Q.X. Liu, Z.Y. Zhang, Analysis of a Delayed SIR Model with Nonlinear Incidence Rate, Discrete Dynamics in Nature and Society, (2008).

[30] M. N'zi, G. Kanga, Global analysis of a deterministic and stochastic nonlinear SIRS epidemic model with saturated incidence rate, Random Oper. Stoch. Equ 24, 1-21. (2016).

[31] J. R.Beddington, Mutual Interference Between Parasites or Predators and its Effect on Searching Efficiency, Journal of Animal Ecology 44, 331-340 (1975).

[32] D.L.DeAngelis, R.A Goldstein, R.V. O'Neill, A Model for Tropic Interaction, Ecology 56, 881-892 (1975).

[33] A.M. Elaiw, S.A. Azoz, Global Properties of a Class of HIV Infection Models with Beddington-DeAngelis Functional Response, Mathematical Methods in the Applied Science 36, 383-394 (2013).

[34] G.Huang, W. Ma, Y. Takeuchi, Global Analysis for Delay Virus Dynamics Model with Beddington-DeAngelis Functional response, Applied Mathematics Letters 24, 1199-1203 (2011).

[35] P. Feng, Analysis of delayed predator-prey model with ratio dependent functional responce and quadratic harvesting, Journal of Applied Mathematics and Computing 44(1), 251-262 (2014).

[36] J. Hale, Theory of Functional Differential Equations, SpringerVerlag, Heidelberg, 1977.

[37] S. Guo, J. Wu, Bifurcation theory of functional differential equations, Springer, 2013.

[38] P. Van den Driessche, J. Watmough, Reproduction Numbers and Sub-Threshold Endemic Equilibria for Compartmental Models of Disease Transmission, Mathematical Biosciences 180, 29-48 (2002).

[39] L. Perko, Differential Equations and Dynamical Systems, Springer, New York, 2000.

[40] S. Wiggins, Introduction to applied nonlinear dynamical systems and chaos, Springer, New York, 2003. 

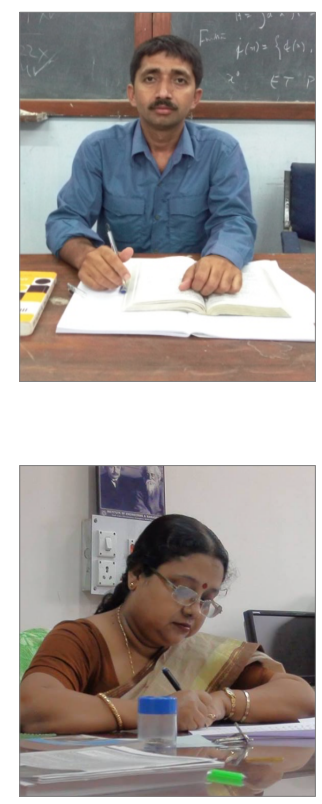

Uttam Ghosh is Assistant Professor of Applied Mathematics, University of Calcutta. His research field includes Fractal geometry, Information theory, Percolation theory, Biomathematics and Fractional Calculus. He has 26 publications in reputed national and international journals.

Susmita Sarkar is Professor of Applied Mathematics, University of Calcutta. Her field of research includes Plasma Dynamics, Biomathematics and Fractional Calculus. She has more than 62 research publications in reputed international journals. Dr. Susmita Sarkar was TWAS Associate and ICTP regular Associate. 